\title{
Lazaroid U83836E protects the heart against ischemia reperfusion injury via inhibition of oxidative stress and activation of PKC
}

\author{
LI-NA LAI ${ }^{*}$, XIAO-JING ZHANG ${ }^{*}$, XIAO-YI ZHANG, LI-HUA SONG, \\ CHUN-HUA GUO, JING-WEN LEI and XIAO-LIANG SONG
}

Department of Pharmacology, Changzhi Medical College, Changzhi, Shanxi 046000, P.R. China

Received June 9, 2015; Accepted March 7, 2016

DOI: $10.3892 / \mathrm{mmr} .2016 .5030$

\begin{abstract}
Oxidative stress has been demonstrated to be important during myocardial ischemia/reperfusion injury (MIRI). The lazaroid U83836E, which combines the amino functionalities of the 21-aminosteroids with the antioxidant ring portion of vitamin $\mathrm{E}$, is a reactive oxygen species scavenger. The aim of the current study was to investigate the effect of U83836E on MIRI and its mechanisms of action. Rat hearts were subjected to 30 min ligation of the left anterior descending coronary artery, followed by $2 \mathrm{~h}$ reperfusion. The results demonstrated that at $5 \mathrm{mg} / \mathrm{kg}, \mathrm{U} 83836 \mathrm{E}$ markedly protected cardiac function in ischemia/reperfusion rat models, decreased the malondialdehyde content and creatinine kinase activity, while increasing superoxide dismutase and glutathione peroxidase activity. Additionally, U83836E significantly decreased the histological damage to the myocardium, reduced the area of myocardial infarction in the left ventricle and modified the mitochondrial dysfunction. Furthermore, U83836E enhanced the translocation of protein kinase $\mathrm{C} \varepsilon$ ( $\mathrm{PKC} \varepsilon$ ) from the cytoplasm to the membrane. However, the cardioprotective effects of U83836E were reduced in the presence of the PKC inhibitor, chelerythrine $(1 \mathrm{mg} / \mathrm{kg})$. Therefore, the results of the present study suggest that U83836E has a potent protective effect against MIRI in rat models through the direct anti-oxidative stress mechanisms and the activation of PKC signaling.
\end{abstract}

\section{Introduction}

Coronary artery disease is currently the leading cause of mortality and morbidity worldwide and acute myocardial infarction is a major cause of death in numerous countries $(1,2)$.

Correspondence to: Professor Xiao-Liang Song, Department of Pharmacology, Changzhi Medical College, 161 Jiefang East Street, Changzhi, Shanxi 046000, P.R. China

E-mail: sx133469399@126.com

*Contributed equally

Key words: U83836E, ischemia/reperfusion, oxidative stress, protein kinase $\mathrm{C}$, mitochondria
The most effective strategy for treating cardiac injury and limiting infarct size is early restoration of coronary blood flow to the ischemic myocardium. While essential, this approach is often associated with functional and structural damage during reperfusion. Although the complex mechanisms of myocardial ischemia/reperfusion injury (MIRI) are not well understood, cardiac dysfunction and cardiomyocyte necrosis during ischemia/reperfusion (I/R) are strongly associated with intracellular $\mathrm{Ca}^{2+}$ overload and oxidative stress (3). Additionally, mitochondrial dysfunction has been proposed to be important to the etiology of MIRI, since the mitochondria act as the source and target of the injury process. Indeed, the mitochondria sustain progressive damage during prolonged cardiac ischemia, and in the process become a major source of excess reactive oxygen species (ROS) generation $(4,5)$. ROS have also been demonstrated to interfere with the $\mathrm{Na}^{+}-\mathrm{Ca}^{2+}$ exchanger and to inhibit $\mathrm{Na}^{+}, \mathrm{K}^{+}$-ATPase activity. Impairment of $\mathrm{Na}^{+}, \mathrm{K}^{+}$-ATPase activity results in $\mathrm{Na}^{+}$overload, with consequent activation of the $\mathrm{Na}^{+}-\mathrm{Ca}^{2+}$ exchanger that may cause intracellular $\mathrm{Ca}^{2+}$ overload and accumulation in mitochondria (6-9). This ultimately results in the formation of mitochondrial permeability transition pores (mPTPs) and breakdown of the mitochondrial membrane potential $(\Delta \Psi \mathrm{m})(10)$. Thus, against this vicious cycle of perpetual damage by ROS and mitochondrial $\mathrm{Ca}^{2+}$ overload, I/R may preserve the structural and functional integrity of the mitochondria and other cell structures to reduce cardiac injury.

The lazaroids are potent scavengers of oxygen free radicals. The lazaroid derivative U83836E combines the amino functionalities of the 21-aminosteroids with the antioxidant ring portion of vitamin E. The antioxidant potency of U83836E is 100 times that of vitamin E (11). Accumulating evidence has demonstrated that U83836E has a variety of pharmacological properties, including anti-neurodegenerative actions (12-14) and the ability to improve blood-brain barrier function (15). Preliminary studies have demonstrated that U83836E $(5 \mathrm{mg} / \mathrm{kg})$ exerts therapeutic effects against hemorrhagic shock and limb I/R in rats (16). The protein kinase $\mathrm{C}(\mathrm{PKC})$ signaling pathways are understood to be involved in MIRI protection (17). In hippocampal neurons and glial cells, PKC is a key enzyme in the signal transduction cascade that leads to superoxide radical generation (18). The potential protective effects of U83836E against I/R injury and preservation of functional integrity of mitochondria remain unclear, in addition to whether this is mediated by PKC activation. The present study was designed to confirm the protective 
effects of U83836E against MIRI in rat models and to identify the possible mechanisms underlying these effects.

\section{Materials and methods}

Experimental animals. The present study was approved by the Animal Care and Use Committees of Changzhi Medical College (Changzhi, China). A total of 120 male Sprague-Dawley (SD) rats (clean grade) of 280-300 $\mathrm{g}$ body weight and 8-10 weeks old were obtained from the Shanxi Medical University Experimental Animal Center (Taiyuan, China). The animal protocol was designed to minimize pain or discomfort to the animals. The animals were acclimatized to laboratory conditions $\left(23^{\circ} \mathrm{C}\right.$, 12-h light/dark cyle, 50\% humidity, ad libitum access to food and water) for 2 weeks prior to experimentation. Intragastric gavage administration was carried out with conscious animals, using straight gavage needles appropriate for the animal size (16 gauge, $10 \mathrm{~cm}$ length, $1.25 \mathrm{~mm}$ ball diameter). All rats were sacrificed by barbiturate overdose (intravenous injection; $150 \mathrm{mg} / \mathrm{kg}$ pentobarbital sodium) for tissue collection.

Establishment of the myocardial I/R injury model. Lead II electrocardiogram was performed for continual monitoring using a BL-420F multi-channels physiologic signal analysis system (Chengdu Taimeng Science and Technology Co., Ltd., Chengdu, China). Tracheotomy was performed and an intubating cannula was connected to a small animal respirator (Taimeng Technology Co., Ltd.). A polyethylene tube (PE 50; BD Biosciences, Franklin Lakes, NJ, USA) filled with heparinized saline was inserted into the left ventricular cavity via the right carotid artery. The left ventricular systolic pressure (LVSP), left ventricular end diastolic pressure (LVEDP), and the maximal rate of increase and decline in left ventricular pressure ( $\pm \mathrm{dp} / \mathrm{dtmax})$ were monitored by the BL-420F signal analysis system. A left thoracotomy was performed from the third to fourth intercostal space and the pericardium was opened. The left anterior descending artery (LAD) was identified and ligated $\sim 2 \mathrm{~mm}$ from its origin by a $5 / 0$ curved suture needle with thread. Ischemia was confirmed by an ST elevation on the ECG and color change to the myocardial tissue of the ischemic area. Following 30-min ischemia, the ligature was loosened and the ischemic myocardium was reperfused for $2 \mathrm{~h}$. At the end of the protocol, the hearts of the rats were excised while the animals were anesthetized.

Experimental design and drug treatment. Male adult SD rats were randomly divided into the following four groups: i) Sham operation group, the LAD coronary artery was crossed by thread without ligation; ii) I/R group, the rats were subjected to LAD occlusion for $30 \mathrm{~min}$ followed by reperfusion for $2 \mathrm{~h}$; iii) U83836E + I/R group, $5 \mathrm{mg} / \mathrm{kg}$ U83836E (PubChem CID 107656; 98\%; Enzo Life Sciences, Inc., Farmingdale, NY, USA) dissolved in physiological saline was administered by tail vein injection $1 \mathrm{~h}$ prior to ischemia; and iv) $\mathrm{CHE}+\mathrm{U} 83836 \mathrm{E}+\mathrm{I} / \mathrm{R}$ group, $1 \mathrm{mg} / \mathrm{kg}$ chelerythrine $\mathrm{PKC}$ inhibitor (CHE; PubChem CID 72311; 99\%; LC Laboratories, Woburn, MA, USA) dissolved in physiological saline was administered by tail vein injection 15 min prior to U83836E administration. The sham and $\mathrm{I} / \mathrm{R}$ groups received an equivalent volume of physiological saline via tail vein injection.
Measurement of the myocardial infarct size. The infarct size was determined by staining with $1 \%$ TTC (99\%; Sigma-Aldrich, St. Louis, MO, USA). Following reperfusion, the heart was rapidly excised from the thorax and washed with physiological saline at $4^{\circ} \mathrm{C}$, then frozen for $10 \mathrm{~min}$ at $-20^{\circ} \mathrm{C}$. The hearts were subsequently transected into five pieces and the slices were incubated in $1 \%$ TTC in phosphate buffer $(\mathrm{pH} 7.4)$ at $37^{\circ} \mathrm{C}$ for $10 \mathrm{~min}$, followed by post-fixation in 10\% formaldehyde (Sinopharm Chemical Reagent Co., Ltd., Shanghai, China) for 15-20 h to enhance the contrast between the stained and unstained TTC tissues. The viable tissue was stained red by TTC, whereas the infarct area remained pale. The area of infarct size (IS) and area of the left ventricle (LV) in each slice were imaged with a scanner (V30; Seiko Epson Corporation, Suwa, Japan) and analyzed by Image-Pro Plus software, version 7.0 (Media Cybernetics, Inc., Rockville, MD, USA). The extent of ischemia in the myocardium was calculated as the ratio of IS/LV.

Histopathological examination. The hearts were fixed in $10 \%$ neutral-buffered formalin and then dehydrated, decalcified and embedded in paraffin (Sinopharm Chemical Reagent Co., Ltd.). Cross-sections (5 $\mu \mathrm{m}$ thick) of the fixed myocardial tissues were cut using a microtome (Leica RM2235; Leica Microsystems GmbH, Wetzlar, Germany). The samples were stained with hematoxylin and eosin (ZSGB-BIO Co., Ltd., Beijing, China), and observed under a light microscope (Olympus BX51; Olympus Corporation, Tokyo, Japan) to analyze the myocardium architecture.

Measurement of creatinine kinase (CK) activity in plasma. Following $2 \mathrm{~h}$ reperfusion, blood samples were collected from the left ventricle and centrifuged at 3,000 $\mathrm{x}$ g for $10 \mathrm{~min}$ to isolate the plasma. Myocardial cellular damage was evaluated by measuring plasma CK activity using assay kits (cat. no. A032; Nanjing Jiancheng Bioengineering Institute, Nanjing, China), according to the manufacturer's protocol.

Evaluation of superoxide dismutase (SOD), glutathione peroxidase (GSH-Px) and malondialdehyde (MDA) in myocardial tissue. Following 2-h reperfusion, the myocardial samples were rinsed and homogenized in deionized water $(1: 10, \mathrm{wt} / \mathrm{vol})$ prior to centrifugation at $3,000 \mathrm{xg}$ for $5 \mathrm{~min}$. The activities of SOD and GSH-Px and the concentration of MDA were quantified using detection kits (cat. nos. A001-1, A005 and A003-1, respectively; Nanjing Jiancheng Bioengineering Institute) according to the manufacturer's protocol.

Isolation of mitochondria. Mitochondrial fractions were isolated by differential centrifugation, as previously described (19). All experiments were performed in cold conditions. Briefly, the LV tissue $(150 \mathrm{mg})$ was first suspended in isolation buffer A (250 mM sucrose; $10 \mathrm{mM}$ Tris- $\mathrm{HCl} ; 1 \mathrm{mM}$ EDTA, pH 7.4; Sinopharm Chemical Reagent Co., Ltd.), finely minced with scissors and then homogenized in deionized water $(1: 10, \mathrm{wt} / \mathrm{vol})$. The homogenates were centrifuged at $1,000 \mathrm{x} \mathrm{g}$ for $10 \mathrm{~min}$, and the supernatant was collected and centrifuged at $1,500 \mathrm{xg}$ for $10 \mathrm{~min}$. The supernatant from this centrifugation was subsequently centrifuged at $10,000 \mathrm{xg}$ for $15 \mathrm{~min}$ to yield the cytosolic fraction. The precipitate, 
corresponding to the mitochondrial fraction, was resuspended in isolation buffer B $(250 \mathrm{mM}$ sucrose; $10 \mathrm{mM}$ Tris- $\mathrm{HCl}$, $\mathrm{pH}$ 7.4). Pellets were maintained on ice prior to $\mathrm{Ca}^{2+}$ and $\Delta \Psi \mathrm{m}$ experiments. The concentration of mitochondrial proteins was measured using bicinchoninic acid assay (Beyotime Institute of Biotechnology, Haimen, China ).

Measurement of mitochondrial $\mathrm{Ca}^{2+}$ concentration $\left(\mathrm{m}\left[\mathrm{Ca}^{2+}\right]\right)$. The suspensions were adjusted to a concentration of $0.5 \mathrm{mg} / \mathrm{ml}$ with buffer $\mathrm{B}$ containing the $\mathrm{Ca}^{2+}$ fluoroprobe Fura2/AM $(10 \mu \mathrm{M})$ and incubated at $37^{\circ} \mathrm{C}$ for $30 \mathrm{~min}$, and then washed to remove the non-bound components. The Fura2/AM Ca ${ }^{2+}$ signals were determined with a F4600 fluorescent spectrophotometer (Hitachi, Ltd., Tokyo, Japan), using an excitation wavelength of $340 \mathrm{~nm}$ and an emission wavelength of $380 \mathrm{~nm}$, as previously described (20). The $\mathrm{Ca}^{2+}$ levels were calculated using the following formula: $\mathrm{K}_{\mathrm{d}} \mathrm{x}\left(\mathrm{R}-\mathrm{R}_{\min }\right) /\left(\mathrm{R}_{\max }-\mathrm{R}\right)$; where $\mathrm{K}_{\mathrm{d}}=314 \mathrm{nmol} / 1, \mathrm{R}$ is the resting state, $\mathrm{R}_{\text {min }}$ is the fluorescence intensity ratio of the $340 \mathrm{~nm}$ and the $380 \mathrm{~nm}$ wavelengths to join the ethylene glycol tetraacetic acid, and $\mathrm{R}_{\max }$ is the fluorescence intensity ratio of the $340 \mathrm{~nm}$ and the $380 \mathrm{~nm}$ wavelengths following Triton X-100 (Sinopharm Chemical Reagent Co., Ltd.) addition.

Measurement of $\Delta \Psi m$. JC-1 dye (Beyotime Institute of Biotechnology) was used to determine the change in $\Delta \Psi_{\mathrm{m}}(21)$. The isolated mitochondria $(0.5 \mathrm{mg} / \mathrm{ml})$ were incubated in $\mathrm{JC}-1$ at $37^{\circ} \mathrm{C}$ for $30 \mathrm{~min}$. The fluorescence intensity was measured using the F4600 spectrofluorometer with $514 \mathrm{~nm}$ excitation and $529 \mathrm{~nm}$ emission wavelengths (Hitachi, Ltd., Tokyo, Japan). The fluorescence intensity ratio of JC-1 polymers and monomers reflects the $\Delta \Psi \mathrm{m}$.

Western blot analysis of PKC. Cytosolic proteins and membrane proteins from the left myocardial samples were extracted using the Membrane and Cytosol Protein Extraction kit (Beyotime Institute of Biotechnology) according to the manufacturer's protocol. Equal protein fractions were separated by electrophoresis on 10\% SDS-PAGE gels. Following transfer to polyvinylidene fluoride (PVDF) membranes, the proteins were probed with a rabbit monoclonal primary antibody against PKCe (1:1,000; ab124806; Abcam, Cambridge, UK) overnight at $4^{\circ} \mathrm{C}$. The PVDF membranes were washed 3 times with Tris-buffered saline containing $0.1 \%$ Tween-20, and incubated with a goat anti-rabbit horseradish peroxidase-conjugated secondary antibody $(1: 2,000$; A0208; Beyotime Institute of Biotechnology) for $2 \mathrm{~h}$ at room temperature. $\beta$-Actin was detected used a rabbit polyclonal antibody (1:5,000; ab119716; Abcam) as a loading control. The proteins were detected with the enhanced chemiluminescence system (Beyotime Institute of Biotechnology) on an ImageQuant LAS 4000 (GE Healthcare Life Sciences, Chalfont, UK). The band intensities were quantified using Image-Pro Plus software.

Statistical analysis. All quantification data are expressed as the mean \pm standard error. For multiple-group comparisons, one-way analysis of variance was performed, followed by the least significant difference t-test. $\mathrm{P}<0.05$ was considered to indicate a statistically significant difference.

\section{Results}

Effects of U83836E on hemodynamics in I/R rats. The LVSP, LVEDP and $\pm \mathrm{dp} / \mathrm{dtmax}$ myocardial functional parameters were measured prior to $\mathrm{I} / \mathrm{R}$, following $30 \mathrm{~min}$ of ischemia, and at 5, 30, 60 and $120 \mathrm{~min}$ of reperfusion (Fig. 1). These parameters were similar among the four groups prior to LAD ligation (Fig. 1). Conversely, the rats in the I/R group exhibited a significant increase in LVEDP, and a significant decrease in LVSP and $\pm \mathrm{dp} / \mathrm{dtmax}$, at $30 \mathrm{~min}$ of ischemia and at 5, 30, 60 and $120 \mathrm{~min}$ of reperfusion (all $\mathrm{P}<0.01$; Fig. 1), as compared with the sham group. Compared with the I/R group, U83836E treatment significantly reduced the LVEDP at $30 \mathrm{~min}$ of ischemia $(\mathrm{P}<0.05)$, and at all time points of reperfusion $(\mathrm{P}<0.05)$. In addition, the LVSP and $\pm \mathrm{dp} / \mathrm{dtmax}$ were significantly increased in the U83836E-treated group following $30 \mathrm{~min}$ of ischemia and at all time points of reperfusion $(\mathrm{P}<0.05$; Fig. 1). These results suggest that pretreatment with U83836E may increase cardiac function following I/R injury. Notably, in the CHE+U83836E+I/R group, the effects of U83836E on the LVSP, LVDEP and \pm dp/dtmax were partly attenuated by CHE treatment (Fig. 1).

U83836E decreases myocardial infarct size after I/R injury. As presented in Fig. 2A and B, I/R induced severe myocardial injury. The infarction size in the I/R group was $61.08 \pm 3.28 \%$ ( $\mathrm{P}<0.001$ vs. the sham group). U83836E treatment significantly reduced the infarction size percentage to $41.60 \pm 3.37 \%$ compared with the $\mathrm{I} / \mathrm{R}$ group $(\mathrm{P}=0.002)$. This result indicates that pretreatment with $\mathrm{U} 83836 \mathrm{E}$ may reduce the size of myocardial infarction in I/R rats. Pretreatment with $\mathrm{CHE}$ abrogated the U83836E-induced decrease in infarct size, and the infarction size was elevated to $52.74 \pm 4.43 \%$ ( $\mathrm{P}=0.062$ vs. $\mathrm{I} / \mathrm{R}$ group; $\mathrm{P}=0.03$ vs. $\mathrm{U} 83836 \mathrm{E}+\mathrm{I} / \mathrm{R}$ group).

Pathological changes in the myocardium. As demonstrated in Fig. 2C, histopathological examination of the myocardium of the sham group revealed tightly aligned cardiac muscle, clear transverse tubules, no evidence of blood vessel enlargement or inflammatory cell infiltration into the interstitial region. However, in the I/R group, edema, confluent coagulation necrosis, neutrophil infiltration and mild inflammation were observed. Rats treated with U83836E demonstrated marked structural improvement, particularly with regard to the degree of myonecrosis, inflammatory cell infiltration and edema compared with the I/R group. In the presence of $\mathrm{CHE}$, U83836E partially failed to preserve the myocardial cellular integrity. There was myofibril loss, the appearance of cracks and an enlarged interstitial region, with minor infiltration of inflammatory cells.

Effects of U83836E on CK activity in plasma. CK is a serum biomarker that reflects myocardial damage. As demonstrated in Fig. 3A, there was a significant increase in $\mathrm{CK}$ activity in the I/R group compared with the sham group $(\mathrm{P}=0.002)$. $\mathrm{CK}$ activity was reduced from $29.06 \pm 3.14 \mathrm{U} / \mathrm{ml}$ in the I/R group to $15.097 \pm 1.88 \mathrm{U} / \mathrm{ml}$ following treatment with $\mathrm{U} 83836 \mathrm{E}(\mathrm{P}<0.01)$. However, CK activity was elevated to $25.28 \pm 2.16 \mathrm{U} / \mathrm{ml}$ by pretreatment with $\mathrm{CHE}(\mathrm{P}=0.235$ vs. $\mathrm{I} / \mathrm{R}$ group; $\mathrm{P}=0.012$ vs. $\mathrm{U} 83836 \mathrm{E}+\mathrm{I} / \mathrm{R}$ group). 

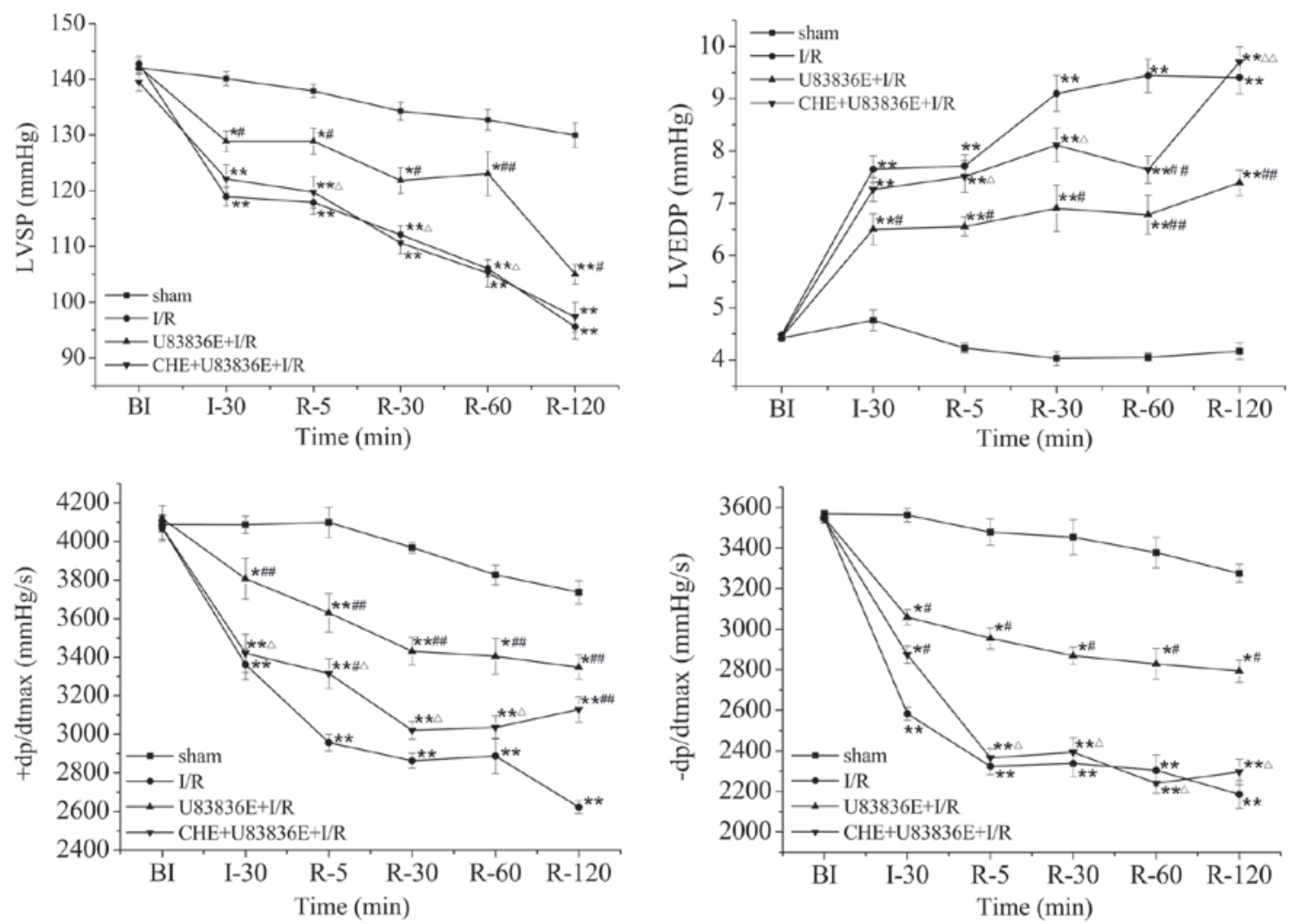

Figure 1. Effects of U83836E on the hemodynamics in rat hearts subjected to $30 \mathrm{~min}$ ischemia followed by $2 \mathrm{~h}$ reperfusion. Values are presented as the mean \pm standard error, $\mathrm{n}=10 .{ }^{*} \mathrm{P}<0.05,{ }^{* *} \mathrm{P}<0.01$ vs. the sham group; ${ }^{*} \mathrm{P}<0.05,{ }^{\# \#} \mathrm{P}<0.01$ vs. the $\mathrm{I} / \mathrm{R}$ group; ${ }^{\Delta} \mathrm{P}<0.05,{ }^{\Delta \Delta} \mathrm{P}<0.01$ vs. the $\mathrm{U} 83836 \mathrm{E}+\mathrm{I} / \mathrm{R}$ group. $\mathrm{BI}$, before ischemia; I, ischemia; R, reperfusion; I/R, ischemia/reperfusion; LVSP, left ventricular systolic pressure; LVEDP, left ventricular end diastolic pressure; +dp/dtmax, maximal rate of increase in left ventricular pressure; -dp/dtmax, maximal rate of increase and decline in left ventricular pressure; CHE, chelerythrine.
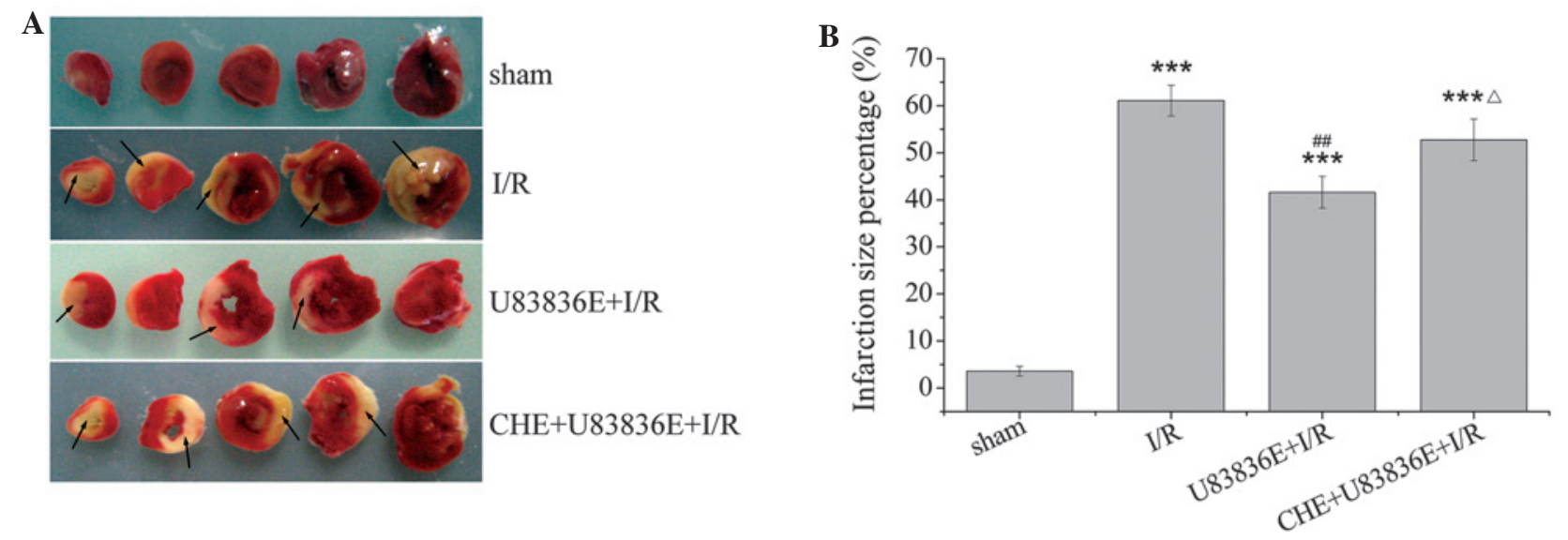

\section{C}

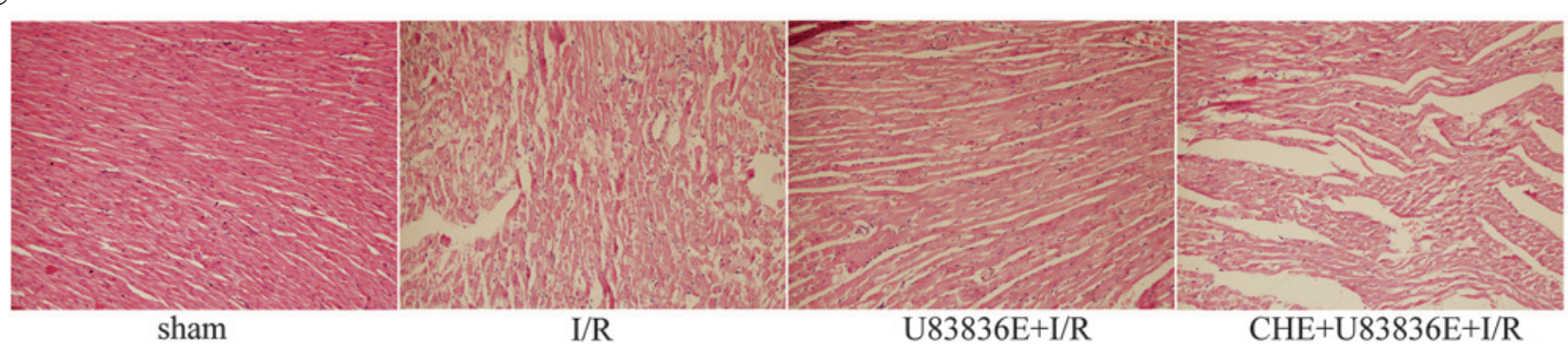

Figure 2. U83836E reduced myocardial I/R injury and the protective effects were abolished by the PKC inhibitor CHE. (A) Slices of the left ventricle were stained by triphenyltetrazolium chloride. Arrows indicate the infarct areas. (B) Percentage infarct area in the left ventricle. (C) Hematoxylin and eosin staining confirmed the protective effects of U83836E $(5 \mathrm{mg} / \mathrm{kg})$ following myocardial I/R. The images are at $\mathrm{x} 200$ magnification. Values are presented as the mean \pm standard error, $\mathrm{n}=8 .{ }^{* * *} \mathrm{P}<0.001$ vs. sham group, ${ }^{\# \#} \mathrm{P}<0.01$ vs. I/R group, ${ }^{\Delta} \mathrm{P}<0.05$ vs. U83836E $+\mathrm{I} / \mathrm{R}$ group. I/R, ischemia/reperfusion; $\mathrm{CHE}$, chelerythrine. 
Effects of U83836E on GSH-Px activity in the myocardial tissue. GSH-Px is regarded as an important antioxidant and free radical scavenger in vivo. As presented in Fig. 3B, the I/R group exhibited a significant decrease in GSH-Px activity compared with the sham group $(\mathrm{P}=0.001)$. U83836E significantly increased the GSH-Px activity in myocardial tissue compared with the I/R group $(\mathrm{P}=0.018)$. No significant difference in GSH-Px activity was observed between the U83836E group and the $\mathrm{CHE}+\mathrm{U} 83836 \mathrm{E}+\mathrm{I} / \mathrm{R}$ group $(\mathrm{P}=0.481)$.

Effects of U83836E on SOD activity and MDA concentration in the myocardial tissue. SOD is an endogenous antioxidant against free radicals and MDA is important indicator of myocardial oxidative damage. Data on these parameters are presented in Fig. 3C and D. Compared with the sham group, the $\mathrm{I} / \mathrm{R}$-induced injury resulted in a significant decrease in SOD activity $(\mathrm{P}=0.002)$ and an increase in the MDA concentration $(\mathrm{P}=0.003)$. The administration of $\mathrm{U} 83836 \mathrm{E}$ resulted in a significant decrease in the MDA concentration $(\mathrm{P}=0.017)$ and increase in the SOD enzyme activity $(\mathrm{P}=0.031)$ compared with the I/R group. Notably, the U83836E-induced changes in MDA concentration $(\mathrm{P}=0.770)$ and SOD activity $(\mathrm{P}=0.819)$ were not significantly altered by $\mathrm{CHE}$ treatment, compared with the $\mathrm{U} 83836 \mathrm{E}+\mathrm{I} / \mathrm{R}$ group.

U83836E prevents the loss of $\Delta \Psi \mathrm{m}$. To further determine the effects of U83836E on mitochondrial function, changes in the $\Delta \Psi \mathrm{m}$ were determined. As demonstrated in Fig. $4 \mathrm{~A}$, the membrane potential in the I/R group was significantly reduced compared with sham group $(2.65 \pm 0.52$ vs. $7.75 \pm 0.51$; $\mathrm{P}=0.003)$, indicating a dissipation of $\Delta \Psi \mathrm{m}$ in response to I/R injury. Compared with I/R group, a significant increase of $\Delta \Psi \mathrm{m}$ was observed following treatment with $\mathrm{U} 83836 \mathrm{E}$ $(\mathrm{P}=0.004)$. Additionally, $\Delta \Psi \mathrm{m}$ was significantly decreased in the $\mathrm{CHE}+\mathrm{U} 83836 \mathrm{E}$ group compared with the U83836E + I/R group $(\mathrm{P}=0.029)$.

U83836E prevents mitochondrial $\mathrm{Ca}^{2+}$ overload. Mitochondrial $\mathrm{Ca}^{2+}$ accumulation is responsible for the cell abnormalities associated with $\mathrm{I} / \mathrm{R}$ injury. As demonstrated in Fig. 4B, compared with the sham group, I/R caused the $\mathrm{m}\left[\mathrm{Ca}^{2+}\right]$ to increase $(\mathrm{P}=0.008)$. The administration of $\mathrm{U} 83836 \mathrm{E}$ resulted in a significant decrease in the $\mathrm{m}\left[\mathrm{Ca}^{2+}\right]$ compared with the $\mathrm{I} / \mathrm{R}$ group $(\mathrm{P}=0.009)$. The $\mathrm{CHE}+\mathrm{U} 83836 \mathrm{E}+\mathrm{I} / \mathrm{R}$ group was observed to be significantly different from the U83836E + I/R group $(\mathrm{P}=0.037)$, but not the $\mathrm{I} / \mathrm{R}$ group $(\mathrm{P}=0.633)$. Altogether, the findings of the present study suggest that pretreatment with $\mathrm{U} 83836 \mathrm{E}$ may alleviate the loss of $\Delta \Psi \mathrm{m}$ and the $\mathrm{m}\left[\mathrm{Ca}^{2+}\right]$ overload in I/R rats. However, the effect of U83836E was diminished by CHE.

U83836E promotes the translocation of PKCE from the cytoplasm to the membrane. PKCE is the most abundant PKC isozyme present in the adult rat cardiac myocytes, and is essential in developing cardioprotection $(22,23)$. Translocation from the cytoplasm to the membrane is crucial for PKC activation. To further explore the relationship between U83836E and PKC $\varepsilon$, the cytosolic and membrane proteins were extracted from the left myocardial samples. As demonstrated in Fig. 5, the PKCE protein levels were significantly increased in the
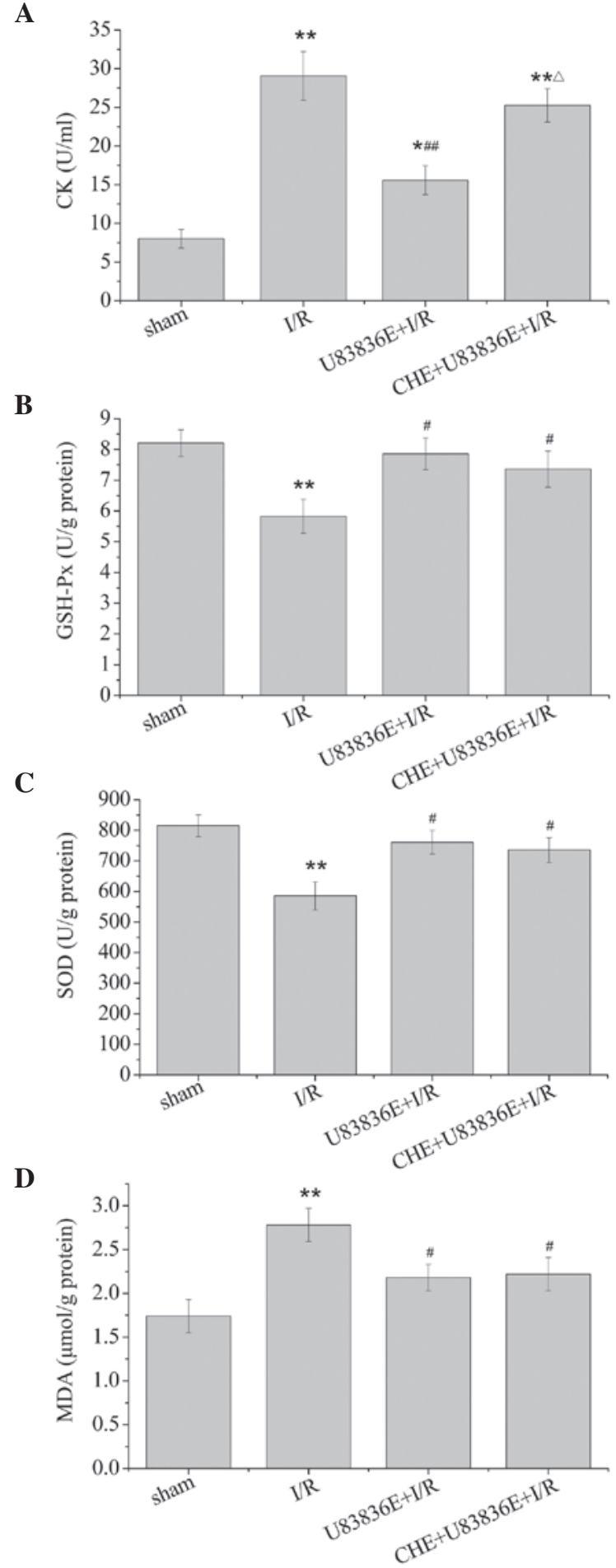

Figure 3. Effects of U83836E on antioxidant and oxidant production in rat hearts subjected to $30 \mathrm{~min}$ of ischemia followed by $2 \mathrm{~h}$ reperfusion. Effect of U83836E ( $5 \mathrm{mg} / \mathrm{kg}$ ) on (A) the CK concentration in the plasma, (B) GSH-Px activity levels in myocardial tissue, (C) SOD activity levels in myocardial tissue and (D) MDA levels in myocardial tissue. Values are presented as the mean \pm standard error, $\mathrm{n}=10$. ${ }^{*} \mathrm{P}<0.05,{ }^{* *} \mathrm{P}<0.01$ vs. sham group; ${ }^{\#} \mathrm{P}<0.05$, ${ }^{\# \#} \mathrm{P}<0.01$ vs. I/R group; ${ }^{\Delta} \mathrm{P}<0.05$ vs. $\mathrm{U} 83836 \mathrm{E}+\mathrm{I} / \mathrm{R}$ group. $\mathrm{CK}$, creatine kinase; GSH-Px, glutathione peroxidase; SOD, superoxide dismutase; MDA, malondialdehyde; I/R, ischemia/reperfusion; CHE, chelerythrine.

$\mathrm{U} 83836 \mathrm{E}+\mathrm{I} / \mathrm{R}$ group compared with the sham and the $\mathrm{I} / \mathrm{R}$ groups $(\mathrm{P}=0.001)$. CHE significantly decreased the translocation of $\mathrm{PKC} \varepsilon$ from the cytoplasm to the membrane compared with the U83836E + I/R group $(\mathrm{P}=0.003)$. 
A
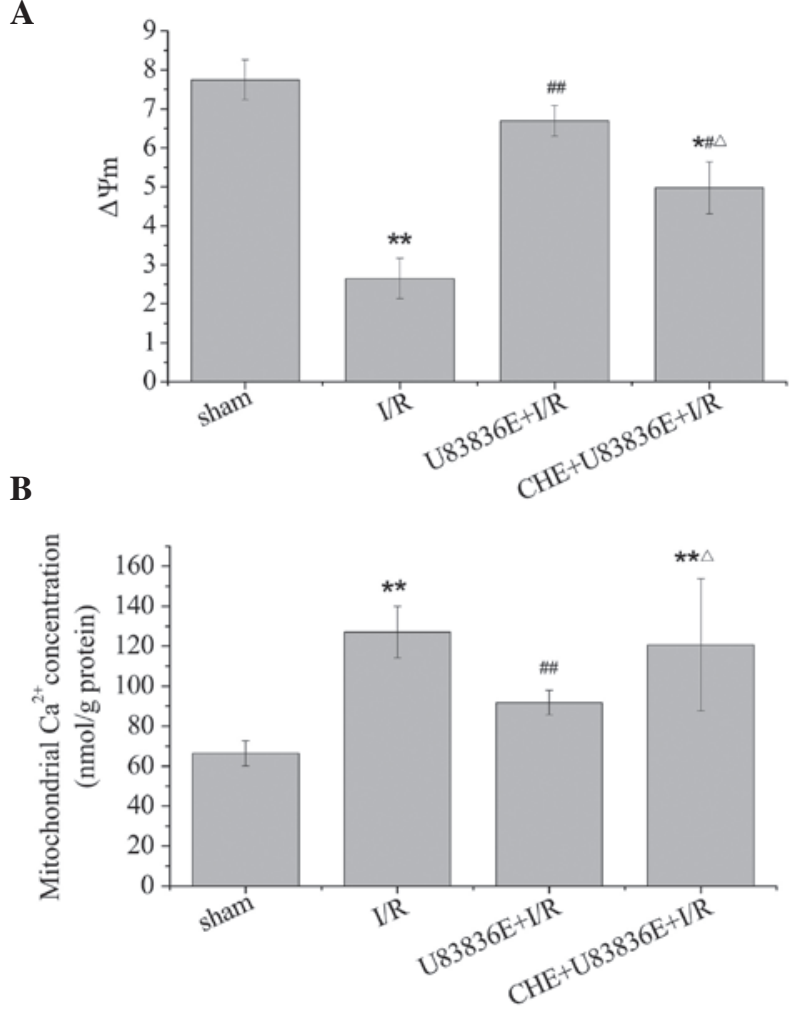

Figure 4. Effects of U83836E on mitochondrial dysfunction in rat hearts subjected to $30 \mathrm{~min}$ of ischemia followed by $2 \mathrm{~h}$ of reperfusion. (A) Changes in $\Delta \Psi \mathrm{m}$ are indicated by JC-1 staining of the mitochondria. (B) Changes in mitochondrial $\mathrm{Ca}^{2+}$ levels are indicated by Fura2/AM staining of the mitochondria. The fluorescence intensity ratio of JC-1 polymers and monomers, and Fura2/AM staining was measured by a spectrofluorometer. Values are presented as the mean \pm standard error. $\mathrm{n}=10$; ${ }^{*} \mathrm{P}<0.05,{ }^{* *} \mathrm{P}<0.01$ vs. sham group; ${ }^{\#} \mathrm{P}<0.05,{ }^{\# \#} \mathrm{P}<0.01$ vs. I/R group; ${ }^{\Delta} \mathrm{P}<0.05$ vs. U83836E $+\mathrm{I} / \mathrm{R}$ group. $\Delta \Psi \mathrm{m}$, mitochondrial membrane potential; I/R, ischemia/reperfusion; CHE, chelerythrine.

\section{Discussion}

Myocardial damage is quickly initiated following ischemia and is enhanced during the reperfusion period. I/R disturbs the balance between the formation and elimination of ROS, resulting in ROS accumulation. The endogenous defense system against ROS, primarily involving the antioxidant enzymes SOD and GSH-Px, is critical to provide a line of defense against oxidative damage under a variety of stress conditions, including I/R injury $(24,25)$. The present study demonstrated that U83836E $(5 \mathrm{mg} / \mathrm{kg})$ significantly increases SOD and GSH-Px activity, and suppresses the formation of the lipid peroxidation product, MDA. These data demonstrate that treatment with U83836E enhances the activity of antioxidant enzymes in myocardial I/R-challenged rats. Myocardial enzyme leakage is indicative of myocardial cell membrane injury. The mass production of oxygen-derived free radicals can result in myocardial cell membrane injury and myocardial enzyme leakage from the myocardial cells. In the current study, it was observed that U83836E treatment significantly inhibited the CK increase during I/R. In addition, treatment with U83836E significantly preserved left ventricular function, as reflected by a significant increase in the indices of contraction (+dp/dtmax), relaxation (-dp/dtmax), LVSP and a decrease in LVEDP in the I/R-insulted rat heart. Furthermore, U83836E

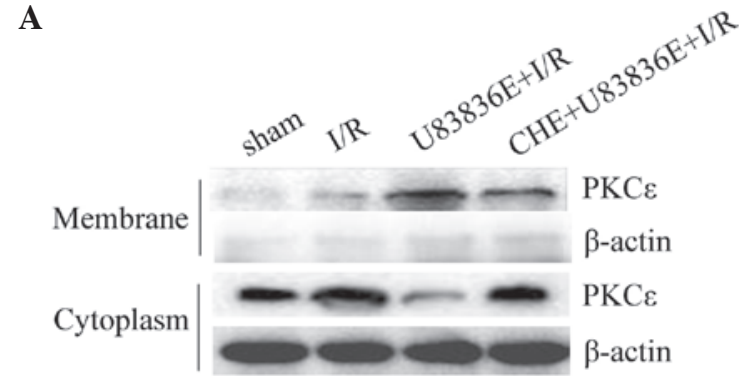

B

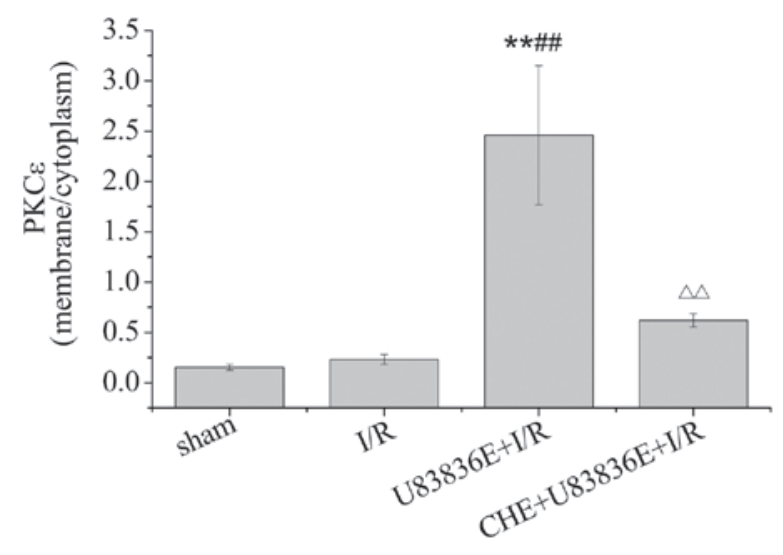

Figure 5. U83836E promoted translocation of PKCe from the cytoplasm to the membrane. (A) Western blot analysis of the subcellular distribution of PKC $\varepsilon$ in the membrane and cytoplasm in rat hearts subjected to $30 \mathrm{~min}$ ischemia followed by $2 \mathrm{~h}$ reperfusion. $\beta$-Actin blots of membrane protein illustrated the high purity of membrane fractions. (B) Quantitative densitometry of PKC $\varepsilon$ ratio in the membrane and the cytoplasm. Values are presented as the mean \pm standard error, $\mathrm{n}=3$. ${ }^{* *} \mathrm{P}<0.01$ vs. sham group, ${ }^{\# \#} \mathrm{P}<0.01$ vs. I/R group, ${ }^{\Delta \Delta} \mathrm{P}<0.01$ vs. U83836E $+\mathrm{I} / \mathrm{R}$ group. I/R, ischemia/reperfusion; CHE, chelerythrine; $\mathrm{PKC} \varepsilon$, protein kinase $\mathrm{C} \varepsilon$.

decreased the histological damage to the myocardium and reduced the area of myocardial infarct of the left ventricle. All these data clearly demonstrate that U83836E exerts protective effects against myocardial I/R injury in rats.

Oxidative stress and $\mathrm{Ca}^{2+}$ overload are the two major factors demonstrated to be associated with the pathology of $\mathrm{I} / \mathrm{R}$ injury. The importance of the mitochondria as targets and mediators of I/R is increasingly recognized. ROS are initially generated at the onset of reperfusion via oxidation of hypoxanthine by xanthine oxidase, and from the mitochondrial electron transport chain reactions. More ROS can be generated through a nicotinamide adenine dinucleotide phosphate (NADPH) reaction catalyzed by NADPH oxidase during extended reperfusion (26). Additionally, intracellular and mitochondrial $\mathrm{Ca}^{2+}$ overload can be caused by various mechanisms, including membrane lipid peroxidation, the opening of voltage-sensitive $\mathrm{Ca}^{2+}$ channels, the $\mathrm{Na}^{+} / \mathrm{Ca}^{2+}$ exchanger, the release of $\mathrm{Ca}^{2+}$ from intracellular endoplasmic and sarcoplasmic reticulum stores, and the attenuation of $\mathrm{Ca}^{2+}$ uptake by the sarcoplasmic reticulum $\mathrm{Ca}^{2+}$-ATPase. $\mathrm{Ca}^{2+}$ alters the inner mitochondrial membrane lipid organization by interacting with the anionic head of cardiolipin, an abundant component of this membrane. These alterations in membrane organization may affect the respiratory chain function and favor monoelectric oxygen reduction to form a superoxide anion at an intermediate step of the respiratory 
chain. ROS generation promotes $\mathrm{Ca}^{2+}$ release from the nearby sarcoplasmic reticulum, leading to $\mathrm{m}\left[\mathrm{Ca}^{2+}\right]$ accumulation that further increases the rate of ROS production (27). These events may be the basis of the findings of the current study regarding the $\mathrm{Ca}^{2+}$-induced $\mathrm{ROS}$ production in I/R injury and may explain the reduction after treatment with U83836E. Under physiological conditions, the inner membrane of the mitochondria is impenetrable to almost all metabolites and ions, and the mPTP exists in a closed conformation. During the stages of reperfusion, $\mathrm{Ca}^{2+}$ overload and excessive ROS production can trigger mPTP opening (28). This opening leads to the dissipation of $\Delta \Psi \mathrm{m}$, the uncoupling of the respiratory chain, the inhibition of ATP production, and mitochondrial swelling and rupture (29). Loss of $\Delta \Psi \mathrm{m}$ can initiate a positive feedback loop by which additional mPTPs open, resulting in further dissipation of $\Delta \Psi \mathrm{m}$ and facilitating persistent $\mathrm{mPTP}$ opening (30). Thus, $\Delta \Psi \mathrm{m}$ is an important parameter in the regulation of mitochondrial function and is used as an indicator of the mPTP status. The current study demonstrated that $\Delta \Psi \mathrm{m}$ depolarization is induced by $\mathrm{I} / \mathrm{R}$ injury and can be significantly alleviated by pretreatment with U83836E. These results suggest that U83836E exerts a protective effect on the mitochondria, inhibiting mPTP opening and maintaining mitochondrial structural integrity during I/R. Overall, U83836E clearly contributes to mitochondrial protection via prevention of $\mathrm{m}\left[\mathrm{Ca}^{2+}\right]$ overload and the loss of $\Delta \Psi \mathrm{m}$ following reperfusion.

The PKC enzymes are central to numerous signal transduction processes, including cardioprotection (31). Activation of PKC was reported to participate in the protective role of ischemia preconditioning, and induced cardioprotection in several animal models $(17,32)$. In addition, PKC protects the mitochondrial ATP-sensitive potassium channel against $\mathrm{Ca}^{2+}$ overload injury in the rat myocardium (33-35). PKCE is an abundant PKC isozyme present in adult rat cardiac myocytes and is essential in cardioprotection development $(22,23)$. In the present study, it was observed that the cardioprotective effect of U83836E was reversed by pretreatment with the CHE PKC inhibitor. The current study additionally observed that $\mathrm{U} 83836 \mathrm{E}$ promotes translocation of $\mathrm{PKC} \varepsilon$ from the cytoplasm to the membrane. However, a notable observation in the present study was that treatment with $\mathrm{CHE}$ following U83836E administration did not change the activities of SOD and GSH-Px, or the MDA concentration. CHE also did not alter the U83836E-induced antioxidative process. Thus, it was hypothesized that U83836E exhibits its antioxidative effect by changing SOD and GSH-Px activities and MDA concentration in a PKC signaling-independent manner. It is assumed that the ROS may act upstream of PKC.

It has been previously demonstrated that ROS can directly activate PKC through sulfhydryl oxidation $(36,37)$. PKC is considered to be a mediator rather than a trigger of this process (38). Therefore, the PKC signaling pathway may not participate in this antioxidative process.

In conclusion, the present investigation suggests that the cardioprotective effect of U83836E is mediated by two different pathways, including the free radical-eliminating mechanisms and the activation of PKC-dependent signaling. On the basis of these observations, therapeutic use of U83836E may have clinical potential for the treatment of cardiac reperfusion injury.

\section{Acknowledgements}

The current study was supported by the Natural Science Foundation of Shanxi Province (no. 2009011055-3), a Key Displine Construction Special Fund Projects grant from the Shanxi Province Colleges and Universities (no. 20131007), and a Science and Technology Innovation Team Project from Changzhi Medical College (no. CX201409).

\section{References}

1. Heidenreich PA, Trogdon JG, Khavjou OA, Butler J, Dracup K, Ezekowitz MD, Finkelstein EA, Hong Y, Johnston SC, Khera A, et al; American Heart Association Advocacy Coordinating Committee; Stroke Council; Council on Cardiovascular Radiology and Intervention; Council on Clinical Cardiology; Council on Epidemiology and Prevention; Council on Arteriosclerosis; Thrombosis and Vascular Biology; Council on Cardiopulmonary; Critical Care; Perioperative and Resuscitation; Council on Cardiovascular Nursing; Council on the Kidney in Cardiovascular Disease; Council on Cardiovascular Surgery and Anesthesia, and Interdisciplinary Council on Quality of Care and Outcomes Research: Forecasting the future of cardiovascular disease in the United States: A policy statement from the American Heart Association. Circulation 23: 933-944, 2011.

2. Nabel EG and Braunwald E: A tale of coronary artery disease and myocardial infarction. N Engl J Med 366: 54-63, 2012.

3. Kloner RA, Przyklenk K and Whittaker P: Deleterious effects of oxygen radicals in ischemia/reperfusion. Resolved and unresolved issues. Circulation 80: 1115-1127, 1989.

4. Chambers JW, Pachori A, Howard S, Iqbal S and LoGrasso PV: Inhibition of JNK mitochondrial localization and signaling is protective against ischemia/reperfusion injury in rats. J Biol Chem 288: 4000-4011, 2013.

5. Becker LB: New concepts in reactive oxygen species and cardiovascular reperfusion physiology. Cardiovasc Res 61: 461-470, 2004.

6. Renlund DG, Gerstenblith G, Lakatta EG, Jacobus WE, Kallman $\mathrm{CH}$ and Weisfeldt ML: Perfusate sodium during ischemia modifies post-ischemic functional and metabolic recovery in the rabbit heart. J Mol Cell Cardiol 16: 795-801, 1984,

7. Reeves JP, Bailey CA, and Hale CC. Redox modification of sodium-calcium exchange activity in cardiac sarcolemmal vesicles. J Biol Chem, 1986,261: 4948-4955.

8. Kim MS and Akera T: $\mathrm{O} 2$ free radicals: Cause of ischemia-reperfusion injury to cardiac $\mathrm{Na}^{+}-\mathrm{K}^{+}$-ATPase. Am J Physiol 252: H252-H257, 1987.

9. Hearse DJ: Stunning: A radical re-view. Cardiovasc Drugs Ther 5: 853-876, 1991 .

10. Tanaka-Esposito C, Chen Q and Lesnefsky EJ: Blockade of electron transport before ischemia protects mitochondria and decreases myocardial injury during reperfusion in aged rat hearts. Transl Res 160: 207-216, 2012.

11. McCall JM, Braughler JM and Hall ED: A new class of compounds for stroke and trauma: Effects of 21-aminosteroid on lipid peroxidation. Acta Anaesthesiol Belg 38: 417-420, 1987.

12. Karlsson J, Love RM, Clarke DJ and Brundin P: Effects of anaesthetics and lazaroid U-83836E on survival of transplanted rat dopaminergic neurons. Brain Res 821: 546-550, 1999.

13. Sayyed SG, Kumar A and Sharma SS: Effects of U83836E on nerve functions, hyperalgesia and oxidative stress in experimental diabetic neuropathy. Life Sci 79: 777 -783, 2006.

14. Wang H, Zheng Z, Gong Y, Zhu B and Xu X: U83836E inhibits retinal neurodegeneration in early-stage streptozotocin-induced diabetic rats. Ophthalmic Res 46: 19-24, 2011.

15. Giese H, Mertsch K and Blasig IE: Effect of MK-801 and U83836E on a porcine brain capillary endothelial cell barrier during hypoxia. Neurosci Lett 191: 169-172, 1995.

16. Labruto F, Song X, Valen G and Vaage J: Lazaroid U-83836E improves tolerance to hemorrhagic shock and limb ischemia and reperfusion in rats and increases cardiac heat shock protein 72. Acad Emerg Med 13: 7-12, 2006.

17. Li H and Lang XE: Protein kinase C signaling pathway involvement in cardioprotection during isoflurane pretreatment. Mol Med Rep 11: 2683-2688, 2015. 
18. Hongpaisan J, Winters CA and Andrews SB: Strong calcium entry activates mitochondrial superoxide generation, upregulating kinase signaling in hippocampal neurons. J Neurosci 24: 10878-10887, 2004.

19. Argaud L, Gateau-Roesch O, Chalabreysse L, Gomez L, Loufouat J, Thivolet-Béjui F, Robert $\mathrm{D}$ and Ovize $\mathrm{M}$ : Preconditioning delays $\mathrm{Ca}^{2+}$-induced mitochondrial permeability transition. Cardiovasc Res 61: 115-122, 2004.

20. Lukács GL and Kapus A: Measurement of the matrix free $\mathrm{Ca}^{2+}$ concentration in heart mitochondria by entrapped fura- 2 and quin2. Biochem J 248: 609-613, 1987.

21. Li D, Liu M, Tao TQ, Song DD, Liu XH and Shi DZ: Panax quinquefolium saponin attenuates cardiomyocyte apoptosis and opening of the mitochondrial permeability transition pore in a rat model of ischemia/reperfusion. Cell Physiol Biochem 34: 1413-1426, 2014

22. Okubo S, Tanabe Y, Fujioka N, Takeda K and Takekoshi N: Differential activation of protein kinase $\mathrm{C}$ between ischemic and pharmacological preconditioning in the rabbit heart. Jpn J Physiol 53: 173-180, 2003.

23. Holzerová K, Hlaváčková M, Žurmanová J, Borchert G, Neckář J, Kolář F, Novák F and Nováková O: Involvement of PKCepsilon in cardioprotection induced by adaptation to chronic continuous hypoxia. Physiol Res 64: 191-201, 2015.

24. Jaeschke $\mathrm{H}$ and Woolbright BL: Current strategies to minimize hepatic ischemia reperfusion injury by targeting reactive oxygen species. Transplant Rev (Orlando) 26: 103-114, 2012.

25. Cheng Z, He W, Zhou X, Lv Q, Xu X, Yang S, Zhao C and Guo L: Cordycepin protects against cerebral ischemia/reperfusion injury in vivo and in vitro. Eur J Pharmacol 664: 20-28, 2011.

26. Zhao ZQ: Oxidative stress-elicited myocardial apoptosis during reperfusion. Curr Opin Pharmacol 4: 159-165, 2004.

27. Limón-Pacheco J and Gonsebatt ME: The role of antioxidants and antioxidant-related enzymes in protective responses to environmentally induced oxidative stress. Mutat Res 674: 137-147, 2009.

28. Baines CP: The mitochondrial permeability transition pore and ischemia-reperfusion injury. Basic Res Cardiol 104: 181-188, 2009.
29. Javadov S and Karmazyn M: Mitochondrial permeability transition pore opening as an endpoint to initiate cell death and as a putative target for cardioprotection. Cell Physiol Biochem 20: 1-22, 2007.

30. Halestrap AP, Clarke SJ and Javadov SA: Mitochondrial permeability transition pore opening during myocardial reperfusion - a target for cardioprotection. Cardiovasc Res 61: $372-385,2004$

31. Budas GR, Churchill EN and Mochly-Rosen D: Cardioprotective mechanisms of PKC isozyme-selective activators and inhibitors in the treatment of ischemia-reperfusion injury. Pharmacol Res 55: 523-536, 2007.

32. Kawamura S, Yoshida K, Miura T, Mizukami Y and Matsuzaki M: Ischemic preconditioning translocates PKC-delta and -epsilon, which mediate functional protection in isolated rat heart. Am J Physiol 275: H2266-H2271, 1998.

33. Wang $Y$ and Ashraf $M$ : Role of protein kinase $C$ in mitochondrial KATP channel-mediated protection against $\mathrm{Ca}^{2+}$ overload injury in rat myocardium. Circ Res 84: 1156-1165,1999.

34. Agudo-López A, Miguel BG, Fernández I and Martínez AM: Role of protein kinase $\mathrm{C}$ and mitochondrial permeability transition pore in the neuroprotective effect of ceramide in ischemia-induced cell death. FEBS Lett 585: 99-103, 2011.

35. Wang C,Hu SM, Xie H, Qiao SG, Liu H and Liu CF: Role of mitochondrial ATP-sensitive potassium channel-mediated PKC- $\varepsilon$ in delayed protection against myocardial ischemia/reperfusion injury in isolated hearts of sevoflurane-preconditioned rats. Braz J Med Biol Res 48: 528-536, 2015.

36. Yang X, Cohen MV and Downey JM: Mechanism of cardioprotection by early ischemic preconditioning. Cardiovase Drugs Ther 24: 225-234, 2010.

37. Otani H: Reactive oxygen species as mediators of signal transduction in ischemic preconditioning. Antioxid Redox Signal 6: 449-469, 2004.

38. Cohen MV and Downey JM: Adenosine: Trigger and mediator of cardioprotection. Basic Res Cardiol 103: 203-215, 2008. 